\title{
Percepciones del tatuaje como expresión cultural en AMBA
}

\section{Perceptions about Tattoo as a cultural expression in AMBA}

\author{
Luisina Aldaz 1 , Ana A. Fuentes Cuiñas², Pablo A. Vailati ${ }^{3}$ y Boris A. Arko ${ }^{4}$
}

${ }^{1}$ Investigadora y profesora de la Universidad Argentina de la Empresa (UADE). Estudiante de la Maestría en Educación. E-mail: 1aldaz@uade.edu.ar

${ }^{2}$ Magíster en Psicología Cognitiva. Doctoranda en Psicología. Investigadora y profesora asociada de la Universidad Argentina de la Empresa (UADE). E-mail: afuentescuinas@uade.edu.ar

${ }^{3}$ Licenciado en Comercialización y Negocios Globales. Docente e Investigador en la Universidad Argentina de la Empresa (UADE). E-mail: pvailati@uade.edu.ar

${ }^{4}$ Estudiante de la Licenciatura en Comercialización. Asistente de investigación en la Universidad Argentina de la Empresa (UADE). E-mail: barko@uade.edu.ar

Los autores agradecen la participación de Juliana Gomis, estudiante de grado de la Universidad Argentina de la Empresa (UADE), en la confección del marco teórico de este artículo.

Universidad Argentina de la Empresa (UADE).

Ciudad Autónoma de Buenos Aires, Argentina.

\section{Resumen}

El tatuaje representa un fenómeno presente desde los comienzos de la historia de la humanidad. A lo largo de los años, supo ocupar diferentes roles asociados a distintos significados y utilidades, y se ha convertido en la actualidad en una forma de expresión de los individuos. En este contexto, el objetivo planteado para este estudio fue descubrir las percepciones del tatuaje como elemento cultural. Para alcanzarlo, se llevó a cabo una investigación descriptiva, de diseño transversal simple, mediante un cuestionario online estructurado y autoadministrado. La muestra se realizó mediante el muestreo bola de nieve y se compuso de 745 individuos que vivían, estudiaban o trabajaban en AMBA (Área Metropolitana de Buenos Aires, Argentina) y tenían entre 18 y 60 años. Como principal resultado de esta investigación se destaca la existencia de fuertes correlaciones entre las principales variables culturales del tatuaje (moda que vino para quedarse; prejuicios en el entorno laboral; expresión cultural). Adicionalmente, fue posible desarrollar un modelo predictivo para la percepción de que el tatuaje representa una expresión cultural, a través de las otras dos variables culturales mencionadas. Por último, se evidencia la existencia de diferencias significativas que permiten reconocer que los individuos más jóvenes presentan un mayor nivel de acuerdo con la idea de que los tatuajes son una moda que vino para quedarse. Palabras clave: tatuaje, cultura del tatuaje, expresión cultural, tatuaje como moda, prejuicios laborales del tatuaje

\section{Abstract}

Tattoo represents a phenomenon present since the beginning of human history. Over the years, it took different roles associated with different meanings and utilities, becoming today a form of expression of individuals. Nowadays, tattoos are understood as fashion ornaments, body art, among others, which integrate a social reality. The individuals who are tattooed do not perceive their body as an organic entity, but as an expressive and 
aesthetic one: they permanently record a story that tells their experiences, feelings and their significant links. Consequently, the tattoo is not a part of the body of the subject, but a synthesis of it. Tattoos are also understood as elements that represent collective identities.

In this context, the objective of this study was to discover the perceptions of tattoos as cultural elements. To achieve this, a descriptive research was carried out, with a simple transversal design, through a structured and self-administered online questionnaire. The sample was collected by snowball sampling and consisted of 745 individuals who live, study or work in AMBA (Metropolitan Area of Buenos Aires, Argentina) and who are between 18 and 60 years old.

As the main results of this research, it is relevant to highlight the existence of strong correlations between the main cultural variables of tattoos (Fashion that will last; Prejudices in the workplace; Cultural expression). Additionally, a multiple linear regression model was formulated to predict the perception that tattoos are a cultural expression through the idea that it represents a fashion that came to stay and the perception that there are prejudices in the work environment regarding the use of tattoos. Finally, there is evidence of significant differences that allow to recognize that younger individuals have a higher level of agreement with the idea that tattoos are a fashion that will last.

These results contribute to previous studies on the subject. Tattoos currently represent elements associated with fashion and culture, which can be reflected in the correlations found between cultural variables, reinforcing previous studies, who add to this panorama the idea that Tattoos constitute a social reality. Moreover, the predicted model found allows to assess the existence of a subculture around the use of tattoo as a cultural expression. Additionally, the importance of the Prejudice variable in the work environment as one of the predictors of the Cultural Expression variable can be evidenced in previous studies that confirm the existence of these prejudices.

Keywords: tattoo, tattoo culture, cultural expression, tattoo as a fashion, tattoo labor prejudices

\section{Introducción}

La modificación del aspecto externo del cuerpo es una costumbre humana y universal: desde la decoración del cuerpo con joyas, vestimentas y peinados hasta mutilaciones, tales como cortar, cercenar o lesionar de forma permanente una parte del cuerpo, entre las que se distinguen las deformaciones esqueléticas, dentarias, ablación del clítoris, circuncisión, escarificación, perforaciones o piercings y tatuajes (Pérez-Cotapos y Cossio, 2006).

A lo largo de la historia, el tatuaje ha estado presente en culturas muy diversas y alejadas en el tiempo y el espacio. Según el contexto, representó diversos significados, a saber: un arte prohibido, informativo, popular, erótico, identificatorio o decorativo; usado con fines curativos o preventivos; alusivo a mitos $\mathrm{y}$ leyendas; entendido como marcas de transición a la adultez o como pruebas de virilidad y coraje; comprendido como signo de realeza en algunas culturas y de barbarie en otras, o utilizado como forma de castigo (Reisfeld, 2005).

En la actualidad, los tatuajes y perforaciones son entendidos como ornamentos de moda, arte corporal, fijación de la personalidad y atracción sexual, e integran una realidad social (Pérez-Cotapos y Cossio, 2006). Los individuos que están tatuados no perciben su cuerpo como una entidad orgánica, sino expresiva y estética: en él graban de manera permanente una historia que cuenta sus experiencias, sentimientos y sus vínculos significativos. En consecuencia, el tatuaje no es una parte más del cuerpo, sino su síntesis (Sastre Cifuentes, 2010).

Según exponen Pérez-Cotapos y Cossio (2006), existen dos tipos de tatuajes: por un lado, el tatuaje permanente es aquel que introduce un pigmento insoluble por debajo de la 
piel a través de una inyección, y por el otro lado, existe una variante de tatuajes temporales o de henna que no necesita de agujas $\mathrm{u}$ otro objeto para perforar la piel, y duran aproximadamente dos semanas. A lo largo del presente texto, se hará referencia al tatuaje permanente como, simplemente, "tatuaje".

En la sociedad contemporánea, el acto voluntario de tatuarse se ha transformado en un gesto individual y en ese sentido, se puede considerar un acto privado; sin embargo, el grafismo trazado en la piel es leído colectivamente y denota la pertenencia a un grupo etario, cultural u otro (Tesone, 2000). Adicionalmente, los tatuajes nacen en contextos culturales que influyen en la decisión del tatuado, por lo que existen razones sociales y culturales que anteceden la decisión personal de tatuarse (Álvarez Licona y Sevilla González, 2002). En este sentido, resulta relevante el estudio sobre las percepciones que una sociedad tiene acerca del tatuaje como símbolo cultural ya que, como se menciona anteriormente, se trata de un elemento cuya función ha evolucionado a lo largo de los años en distintas culturas (Reisfeld, 2005; Pérez-Cotapos y Cossio, 2006).

En este contexto, se propuso desarrollar una investigación cuantitativa con el propósito de analizar el fenómeno del tatuaje como elemento cultural, atendiendo a las diferencias perceptuales de distintos segmentos y analizando las relaciones entre distintas variables pertinentes a la temática.

\section{Marco teórico}

\section{El tatuaje como moda}

Gil Mártil (2009) considera que algo es moda cuando la mayor parte de los integrantes de un grupo así lo cree. Si bien no es necesario que todos los miembros del grupo lo adopten, es importante que consideren normal que otros lo hagan. En este sentido, algunos individuos que se autodenominan "artistas", y no tatuadores, consideran que la práctica del tatuaje se ha convertido en una moda (Reisfeld, 2005).

Una investigación realizada con pacientes en el Centro de Dermatología Láser del Hospital General de Massachusetts, antes o durante las primeras etapas de la aplicación de un tratamiento láser de remoción de tatuajes, vislumbró que casi la mitad de los pacientes (42 \%) había sentido una fuerte presión por parte de sus pares para tomar las decisiones relacionadas con el tatuaje (Armstrong, Stuppy, Gabriel y Anderson, 1996). A su vez, el $61 \%$ se había interesado en tatuarse con un grupo de amigos, el $58 \%$ había contado con la presencia de dos o más personas antes de tatuarse y el $65 \%$ manifestó que alguien de sus grupos cercanos se había tatuado al mismo tiempo que ellos. En suma, el grupo arrastra a los individuos hacia cierta moda (Gil Mártil, 2009), debido a que el aspecto del contagio o las conductas imitativas confieren la tranquilidad que genera estar haciendo lo mismo que otros (Reisfeld, 2005).

Los resultados de una encuesta telefónica realizada en Estados Unidos a nivel nacional por Pew Research Center (2010) dejan vislumbrar el aumento de la tendencia de la posesión de tatuajes generación tras generación: casi cuatro de cada diez millennials estadounidenses tienen, por lo menos, un tatuaje; por su parte, el $32 \%$ de quienes pertenecen a la generación X (aquellos nacidos entre 1965 y 1980) está tatuado, y solo el $15 \%$ de los baby boomers (nacidos entre 1946 y 1964) y el $6 \%$ de los pertenecientes a la generación silenciosa (nacidos antes de 1946) lo está. En otras palabras, existe una fuerte asociación entre la edad de los individuos y la presencia de tatuajes, siendo las personas más adultas menos proclives a portar uno (Dean, 2010).

\section{El tatuaje como forma de expresión de identidad y cultural}

La cultura es el conjunto de creencias, valores y costumbres aprendidos por un individuo, que sirven para dirigir su comportamiento como miembro de una sociedad 
particular (Schiffman y Kanuk, 2010). Por su parte, el significado de subcultura o microcultura abarca los valores o símbolos de un grupo restringido de individuos, definidos de acuerdo a diversas variables, tales como la edad, religión, etnicidad o alguna otra subdivisión del todo. En efecto, la cultura de un individuo es lo que le da el sentido de identidad (Blackwell, Miniard y Engel, 2002).

Bajo la misma mirada de la relación cultura-identidad, Reisfeld (2005) explica que en el acto de tatuarse está en juego la identidad total del sujeto. Él mismo encarna concretamente los códigos, las tradiciones y los valores que fundamentan su existencia como miembro de un grupo (es decir, miembro de una subcultura). Tal como explican Álvarez Licona y Sevilla González (2002), en los casos en que el tatuaje de un individuo representa no solamente a su persona sino a un grupo, se cohesiona con el grupo; es decir, los tatuajes de los distintos individuos parecerían seguir una línea que demuestra que no solamente representan a sus portadores individualmente, sino al grupo al que pertenecen.

La realización de un tatuaje es una decisión que viene acompañada de expectativas internas de autoidentidad (Armstrong, Stuppy, Gabriel y Anderson, 1996). En otras palabras, un tatuaje es una expresión gráfica de una producción psíquica del sujeto, el cual tiene un valor metonímico del mundo interior, pero no necesariamente metafórico (Tesone, 2000). El tatuaje revela un rasgo sustancial del hombre: la necesidad de procesar y significar sus propias vivencias y experiencias, y de darles alguna forma de expresión (Reisfeld, 2005). No obstante, el tatuaje se entiende también como un elemento que representa cierta identidad colectiva (Álvarez Licona y Sevilla González, 2002).

La idea de reflejar la propia identidad a través del arte corporal también fue apoyada por un estudio realizado por Armstrong y Murphy (1997) en escuelas secundarias de ocho estados de Estados Unidos, el cual reportó que el $81 \%$ de los adolescentes había obtenido su tatuaje con el propósito de ser ellos mismos y no de impresionar a otros, mientras que el $50 \%$ consideraba que sus tatuajes los hacían sentir únicos y especiales. La piel le otorga al adolescente una superficie que le permite, por medio del dibujo sobre ella, darle un sentido al caos de lo irrepresentable de sus propias transformaciones (Tesone, 2000), es decir, el cuerpo es el espacio que permite que a través de prácticas como el tatuaje, se canalice una amplia gama de situaciones inconscientes (Reisfeld, 2005).

Por otra parte, la idea de remover un tatuaje también está relacionada con la identidad del portador, dado que surge ante la motivación interna de disociarse de las experiencias del pasado y mejorar el concepto o la imagen de uno mismo (Armstrong, Stuppy, Gabriel y Anderson, 1996). Los mismos autores sostienen que las razones para comenzar un proceso de remoción de tatuajes son: los cambios positivos a nivel personal, la madurez y el deseo de tener una autoimagen más saludable.

\section{El tatuaje y los prejuicios en el ámbito laboral}

La presencia de tatuajes visibles en el cuerpo puede reducir considerablemente la posibilidad de obtener empleo, sobre todo en los sectores de belleza, venta minorista, oficinas y hospitalidad, donde dicha posibilidad se puede ver minimizada un $70 \%$ o más (Bekhor, Bekhor y Gandrabur, 1995). Esto mismo es apoyado por Dean (2010), quien expone que los tatuajes visibles son considerados como inapropiados para los cuellos blancos, es decir, para personas que desarrollan tareas semiprofesionales o profesionales; pero como apropiados para los cuellos azules, es decir, aquellos que realizan tareas manuales.

Sin embargo, los prejuicios de los tatuajes en el ámbito laboral dependen ampliamente del sector que se considere. Por ejemplo, mientras que en el ámbito de la construcción y los servicios públicos, menos de la mitad 
de los empleadores se deja influenciar por los tatuajes a la hora de reclutar personal, en el sector de belleza, hospitalidad, venta minorista y oficinas la decisión de las personas encargadas de contratar personal se ve considerablemente afectada por la presencia o ausencia de tatuajes en el futuro empleado, siendo menos propensos a emplear personas que cuenten con alguno (Bekhor, Bekhor y Gandrabur, 1995). La presencia de tatuajes en personas que trabajan en el sector financiero proyecta una imagen de poca profesionalidad y el prejuicio de que no son confiables (Dean, 2010). En el sector de los restaurantes y servicios de comida, los empleadores consideran como un aspecto desfavorable la presencia de tatuajes en los futuros empleados; es decir, existe una preferencia por contratar gente sin tatuajes visibles en relación a alguien que sí los tiene (Brallier, Maguire, Smith y Palm, 2011). Por su parte, aquellos que trabajan en el sector de la salud y cuentan con tatuajes visibles son percibidos como poco higiénicos (Dean, 2010).

No obstante, estas percepciones están fuertemente relacionadas con la edad de las personas. Los más jóvenes tienen una percepción más favorable con respecto a los portadores de tatuajes en comparación con los de más edad, quienes consideran menos inteligentes, más rebeldes, con menos encanto $\mathrm{y}$ menos honestos a las personas tatuadas que a las que no lo están (Dean, 2010).

Según un estudio realizado por Foltz (2014), la mayoría de los estudiantes universitarios millennials, tanto tatuados como no tatuados, percibe que la búsqueda laboral luego de terminar sus estudios universitarios será más difícil para aquellos que posean un tatuaje en comparación con los que no tengan ninguno. En consecuencia, la mayoría de los estudiantes tiene en cuenta su futuro empleo a la hora de elegir el lugar del cuerpo para un tatuaje. De igual modo, quienes ya cuentan con un tatuaje deciden tapárselo para las entrevistas laborales o reuniones de trabajo formales. Por otra parte, el estudio también revela que los millennials que tienen un tatuaje deben tener en cuenta que no solo puede generar un impacto negativo en el proceso de conseguir un trabajo, sino que, una vez conseguido el puesto laboral, podría causar interferencias negativas con compañeros de trabajo de mayor edad, debido a los prejuicios que vienen asociados con el tatuaje.

Por último, es importante resaltar que un estudio realizado por Armstrong et al. (2008) en diferentes centros de remoción de tatuajes en Arizona, Colorado, Massachusetts y Texas, verificó que el $38 \%$ de quienes deciden quitarse su tatuaje lo hace debido a un nuevo trabajo o una nueva carrera.

\section{Objetivos}

Como objetivo general de esta investigación, se planteó descubrir las percepciones del tatuaje como elemento cultural. Para alcanzarlo, se establecieron los siguientes objetivos específicos:

1) Determinar la existencia y carácter de las relaciones entre ciertas variables asociadas a la importancia e incidencia del tatuaje en la cultura.

2) Identificar la existencia de diferencias perceptuales entre grupos etarios en función de las variables asociadas a la cultura del tatuaje.

\section{Metodología}

\section{Procedimiento}

La presente investigación se llevó a cabo a partir de un diseño descriptivo transversal simple, el cual se caracteriza por descubrir relaciones existentes entre dos o más variables, permitiendo al investigador realizar predicciones incipientes (Hernández Sampieri, Fernández Collado y Baptista Lucio, 2006). La recolección de datos se realizó a partir de un cuestionario online estructurado y autoadministrado, que los participantes recibieron por medios electrónicos. 
Una vez que el período de trabajo de campo finalizó, se codificaron las variables y se realizaron análisis de correlaciones bivariadas, análisis de regresión lineal múltiple y ANOVAs a través del programa estadístico SPSS en su versión 20.

\section{Instrumento}

Como instrumento de medición se desarrolló un cuestionario online estructurado, que fue respondido por los participantes en forma autoadministrada. En primer lugar, se incluyeron preguntas sociodemográficas que permitieron perfilar la muestra (edad, género, situación laboral, entre otras). Por último, se incluyeron preguntas relacionadas con la temática(percepciones y opiniones acera del tatuaje como expresión cultural), que permitieron realizar el análisis presentado en este artículo.

Para analizar la validez del instrumento, se realizó una evaluación sistemática del contenido de las preguntas de la escala. Adicionalmente, se analizó la validez de constructo de carácter nomológico, evaluando la relación entre los resultados obtenidos a través de la escala con constructos teóricos y resultados previos expuestos en investigaciones anteriores. Por último, se abordó la confiabilidad de consistencia interna, obteniendo un alfa de Cronbach de .51 .

\section{Participantes}

El método de muestreo utilizado fue el de bola de nieve, a través del cual se les pidió a los encuestados que reenviaran el formulario a sus conocidos, con el propósito de obtener la mayor cantidad posible de respondentes.

La muestra estuvo compuesta por 745 individuos que vivían, estudiaban o trabajaban en AMBA (Área Metropolitana de Buenos Aires) y tenían entre 18 y 60 años $(M=23.94, D E=$ 6.96).

\section{Consentimiento informado}

Debido al hecho de que la encuesta fue autoadministrada, se incluyó al inicio una explicación de los objetivos de esta investigación y una pregunta filtro solicitando la aceptación a formar parte de la muestra de este estudio. Quienes eligieran no participar, finalizarían la encuesta y quedarían excluidos de la investigación.

\section{Resultados}

A fin de determinar la existencia y carácter de las relaciones entre las variables asociadas a la cultura del tatuaje ("Me parece que el tatuaje es una moda que vino para quedarse" o Moda que vino para quedarse; "Me parece que existen prejuicios en el entorno laboral respecto al tatuaje" o Prejuicios en el entorno laboral; "Creo que el tatuaje es una expresión cultural” o Expresión cultural) se realizó una prueba de correlaciones bivariadas, obteniéndose los resultados que se observan en la Tabla 1.

Tabla 1

Correlaciones de Pearson entre las variables culturales.

\begin{tabular}{c|ccc}
$\mathbf{n}=\mathbf{7 4 5}$ & $\begin{array}{c}\text { Moda que vino } \\
\text { para quedarse }\end{array}$ & $\begin{array}{c}\text { Expresión } \\
\text { cultural }\end{array}$ & $\begin{array}{c}\text { Prejuicios en el } \\
\text { entorno laboral }\end{array}$ \\
\hline Moda que vino para quedarse & 1 & $.295^{* *}$ & $.210^{* *}$ \\
Expresión cultural & $0.295^{* *}$ & 1 & $.279 * *$ \\
Prejuicios en el entorno laboral & $0.210^{* *}$ & $.279 * *$ & 1
\end{tabular}

** La correlación es significativa en el nivel .01 (bilateral).

Fuente: Elaboración propia. 
Posteriormente, se realizó un análisis de regresión lineal múltiple aplicando las variables Moda que vino para quedarse $\left(\mathrm{X}_{1}\right) \mathrm{y}$ Prejuicios en el entorno laboral $\left(\mathrm{X}_{2}\right)$ como elementos predictivos de la variable Expresión cultural $(\mathrm{Y})$. Se encontró que los predictores logran explicar un $13.6 \%$ de la varianza de la variable dependiente: $R^{2}=.13, \mathrm{~F}(1,743)$ $=58.630, p<.001$. Tanto Moda que vino para quedarse $(\beta=.248, p<.000)$ como Prejuicios en el entorno laboral $(\beta=.227, p<.001)$ muestran efectos sobre la variable dependiente, constituyéndose la siguiente regresión lineal múltiple: $\mathrm{Y}=1.820+.248 \mathrm{X}_{1}+.227 \mathrm{X}_{2}$

Finalmente, se realizaron análisis de varianza a fin de determinar la existencia de diferencias entre los grupos etarios en función de las variables culturales, encontrándose los siguientes resultados:

Edad/Moda que vino para quedarse: El estadístico de Levene $(.444, p=.505)$ permite asumir la igualdad de varianzas entre los grupos. El análisis de la varianza permite afirmar la existencia de diferencias significativas entre los grupos de la variable Edad en función de la variable Moda que vino para quedarse: $\mathrm{F}(1,743)=7.237, p<.05, \eta^{2}=.010$. Entonces, podría afirmarse que los individuos entre 18 y 25 años $(M=3.16, D E=1.187)$ presentan un mayor nivel de acuerdo con la idea de que los tatuajes son una moda que vino para quedarse frente a los encuestados entre 26 y 60 años $(M=2.9, D E=1.185)$.

Edad/Expresión cultural: El estadístico de Levene (8.326, $p=.004)$ no permite asumir la igualdad de varianzas entre los grupos y el análisis de la varianza no permite afirmar la existencia de diferencias significativas entre los grupos de la variable Edad en función de la variable Expresión cultural: $\mathrm{F}(1,743)=.809$, $p=.369, \eta^{2}=.001$.

Edad/Prejuicios en el entorno laboral: $\mathrm{Si}$ bien el análisis de la varianza pareciera indicar la existencia de diferencias entre los grupos $\left[\mathrm{F}(1,743)=22.357, p<.001, \eta^{2}=.029\right]$, el estadístico de Levene $(17.236, p=.000)$ no permite asumir la igualdad de varianzas, por lo que el resultado del ANOVA no es confiable. Entonces, podría afirmarse que no hay suficiente evidencia para identificar la existencia de diferencias significativas entre los grupos etarios en función de la variable dependiente.

\section{Limitaciones y alcance}

La principal limitación de esta investigación es la metodología de muestreo utilizada que, al no ser un método probabilístico, impide la generalización de los resultados a la población total en cuestión. Además, existe una mayor cantidad de respuestas de jóvenes, lo que se evidencia en la distribución de la variable edad, presentando una media de 23.94 años y un desvío estándar de 6.96.

En cuanto a la fundamentación teórica de la temática, se ha descubierto que no existe gran cantidad de artículos científicos que aborden el estudio del tatuaje como elemento cultural desde una perspectiva cuantitativa. En este sentido, se ha realizado una selección de las investigaciones previas más relevantes y se han incluido también ciertos trabajos teóricos o de naturaleza cualitativa que ofrecen un antecedente para los resultados del presente estudio.

En cuanto al alcance de esta investigación, debe considerarse que se toma como población a AMBA (Área Metropolitana de Buenos Aires). Adicionalmente, aunque existen dos tipos de tatuajes, los permanentes y los temporales o de henna (Pérez-Cotapos y Cossio, 2006), a lo largo del presente texto se refiere al tatuaje permanente como simplemente tatuaje, y no se aborda el uso de tatuajes temporales o de henna.

\section{Conclusiones y discusión}

Los tatuajes representan en la actualidad elementos asociados con la moda y la cultura, lo que puede reflejarse en las correlaciones halladas entre las variables culturales, reforzando las afirmaciones de Pérez-Cotapos y Cossio (2006), quienes agregan a este pano- 
rama la idea de que los tatuajes constituyen una realidad social.

La variable Expresión cultural presentó importantes correlaciones con las variables Moda que vino para quedarse (correlación del $29.5 \%$ ) y Prejuicios en el entorno laboral (correlación del $27.9 \%$ ). En este sentido, la regresión lineal múltiple que se formuló con estas variables demuestra que es posible identificar un modelo predictivo de la utilización del tatuaje como una expresión cultural a partir de la idea de que representa una moda que vino para quedarse y la percepción de que existen prejuicios en el ambiente laboral en cuanto al uso de tatuajes.

Este modelo podría evidenciar la existencia de una subcultura en torno al uso del tatuaje como expresión cultural (siguiendo el concepto de subcultura de Schiffman y Kanuk, 2010), evidenciada por un grupo restringido de individuos que consideran que el tatuaje es una moda no pasajera, pero reconocen la existencia de prejuicios en el entorno laboral (probablemente provenientes de individuos que no forman parte de esta subcultura). Esto concuerda con la descripción propuesta por Armstrong, Stuppy, Gabriel y Anderson (1996), quienes informan en su estudio que casi la mitad de los participantes se vieron influenciados por sus pares a la hora de tomar decisiones relacionadas con el tatuaje, considerando que el $61 \%$ se había interesado en tatuarse con un grupo de amigos. Asimismo, en línea con lo expuesto por Gil Mártil (2009) y Reisfeld (2005), un grupo cultural (o una subcultura) tiene la capacidad de arrastrar a los individuos hacia cierta moda, debido a que se presentan conductas imitativas entre los individuos.

Adicionalmente, la importancia de la variable Prejuicios en el entorno laboral como una de las predictoras de la variable Expresión cultural, puede evidenciarse en estudios anteriores que ratifican la existencia de estos prejuicios (Bekhor, Bekhory Gandrabur, 1995; Dean, 2010; Brallier, Maguire, Smithy Palm, 2011; Foltz, 2014). Resulta relevante también considerar los resultados del estudio de Armstrong et al. (2008), quienes exponen que el $38 \%$ de los participantes de su investigación decidieron remover su tatuaje debido a un nuevo trabajo o una nueva carrera.

Con respecto a la idea de que los tatuajes son una moda que vino para quedarse y la percepción de que existen prejuicios en el entorno laboral relacionados con su uso, la correlación es menor que las anteriores, pero permite entender que existe una considerable relación entre las variables (correlación del $21 \%$ ). En este sentido, el concepto de moda explicado por Gil Mártil (2009), es decir, la consideración de que algo es moda cuando la mayoría en un grupo así lo cree, se relaciona con el hecho de que los distintos grupos culturales poseen diferentes consideraciones en cuanto a qué se considera aceptable a la hora de ocupar un puesto de trabajo.

El análisis de la varianza permitió descubrir que los individuos más jóvenes (entre 18 y 25 años) presentan un mayor nivel de acuerdo con la idea de que los tatuajes son una moda que vino para quedarse, frente a los encuestados de mayor edad (entre 26 y 60 años), lo que concuerda con las ideas expuestas por Tesone (2000), quien afirma que el tatuaje denota la pertenencia a un grupo etario y cultural, y Dean (2010), quien sostiene la existencia de una fuerte asociación entre la edad de un individuo y la posesión de tatuajes. Siguiendo con esta línea, una investigación realizada en Estados Unidos demuestra el aumento de la tendencia de la posesión de tatuajes generación tras generación (Pew Research Center, 2010).

Por último, pero no de menor relevancia, los resultados de esta investigación demostrarían la ausencia de diferencias significativas entre grupos etarios en función de la creencia de que los tatuajes representan una expresión cultural y la percepción de que existen prejuicios en el entorno laboral relacionados con la posesión de tatuajes. Esta última afirmación no concuerda con lo expuesto por Dean (2010), quien expone que las percepciones 
relacionadas con los prejuicios sobre el tatuaje estarían fuertemente relacionadas con la edad de las personas.

Cabe destacar que, como se mencionó anteriormente, el presente estudio se caracteriza por una mayor cantidad de respondentes jóvenes, por lo que es probable que las respuestas representen más fielmente a este segmento de la sociedad. Se necesitan estudios posteriores con métodos equitativos de muestreo que permitan conseguir proporciones que representen la distribución de edades de la población estudiada.

\section{Referencias bibliográfícas}

Álvarez Licona, N. E. y Sevilla González, M. L. (2002). Semiótica de una práctica cultural: el tatuaje. Cuicuilco, 9(25), 0. https://www. redalyc.org/articulo.oa?id $=351 / 35102512$

Armstrong, M. y Murphy, K. (1997). Tattooing: another adolescent risk behavior warranting health education. Applied Nursing Research: ANR, 10(4), 181-189. https://doi.org/10.1016/ s0897-1897(97)80560-5

Armstrong, M., Roberts, A., Koch, J., Saunders, J., Owen, D.y Anderson, R. (2008). Motivation for contemporary tattoo removal: a shift in identity. Archives of Dermatology, 144(7), 879-884. doi: 10.1001/archderm.144.7.879.

Armstrong, M., Stuppy, D., Gabriel, D.y Anderson, R. (1996). Archives of Dermatology, 132(4), 412-416.

Bekhor, P., Bekhor, L.y Gandrabur, M. (1995). Employer attitudes toward persons with visible tattoos. Australasian Journal of Dermatology, 36(2), 75-77. https://doi. org/10.1111/j.1440-0960.1995.tb00936.x

Blackwell, R. D., Miniard, P. y Engel, J. (2002). Comportamiento del consumidor. (G. Sánchez, Trad.) México D.F., México: Thomson.
Brallier, S., Maguire, K., Smith, D. y Palm, L. (2011). Visible Tattoos and Employment in the Restaurant Service Industry. International Journal of Business and Social Science, 2(6), 72-76.

Dean, D. H. (2010). Consumer perceptions of visible tattoos on service personnel. Managing Service Quality: An International Journal, 20(3), 294-308. https://doi. org/10.1108/09604521011041998

Foltz, K. A. (2014). The millennial's perception of tattoos: self expression or business faux pas? College Student Journal, 48(4), 589-602.

Gil Mártil, V. A. (2009). Coolhunting: el arte y la ciencia de descifrar tendencias. Conozca hoy lo que sus clientes demandarán mañana. Barcelona, España: Empresa Activa.

Hernández Sampieri, R., Fernández Collado, C. y Baptista Lucio, P. (2006). Metodología de la Investigación. México: Mac Graw-Hill.

Pérez-Cotapos, M. L. y Cossio, M. L. (2006). Tatuajes y perforaciones en adolescentes. Revista Médica de Chile, 134(10), 1322-1329.

Pew Research Center (2010). Millennials: Confident. Connected. Open to Change. Estados Unidos: Pew Research Center.

Reisfeld, S. (2005). Tatuajes: una mirada psicoanalítica. Buenos Aires, Argentina: Paidós.

Sastre Cifuentes, A. (2011). Cuerpos que narran: la práctica del tatuaje y el proceso de subjetivación. Diversitas: Perspectivas en Psicología, 7(1), 179-191. https://www.redalyc.org/ articulo.oa? $\mathrm{id}=679 / 67922583013$

Schiffman, L. G. y Kanuk, L. L. (2010). Comportamiento del consumidor. (V. d. Alba Ramírez, Trad.). Naucalpan de Juárez, México: Pearson Educación.

Tesone, J. E. (2000). El tatuaje y el escudo de Perseo. Revista de la Asociación Argentina de Psicología y Psicoterapia de Grupo, 23(2), 179-195.

Recibido: 27 de julio de 2019 Aceptado: 3 de diciembre de 2020 